\title{
Combined analysis of HLA class I, HLA-E and HLA-G predicts prognosis in colon cancer patients
}

E C M Zeestraten ${ }^{1,4}$, M S Reimers ${ }^{1,4}$, S Saadatmand ${ }^{1}$, I J Goossens-Beumer ${ }^{2}$, J-W T Dekker ${ }^{1}$, G J Liefers ${ }^{1}$, P J van den Elsen ${ }^{2,3}$, C J H van de Velde ${ }^{1}$ and P J K Kuppen ${ }^{*} 1$

${ }^{1}$ Department of Surgery, Leiden University Medical Center, Albinusdreef 2, 2333 ZA Leiden, The Netherlands; ${ }^{2}$ Department of Immunohematology and Blood Transfusion, Leiden University Medical Center, Albinusdreef 2, 2333 ZA Leiden, The Netherlands and ${ }^{3}$ Department of Pathology, VU University Medical Center, de Boelelaan 1117, 1081 HV Amsterdam, The Netherlands

\section{Correction to: British Journal of Cancer (2014) 110, 459-468; doi:10.1038/ bjc.2013.696 \\ J Goossens-Beumer should have been included as the fourth author; the full and correct author listing is now given above.}

Upon publication of this paper in Volume 110 earlier this year, the authors noted they had omitted Inès J Goossens-Beumer from the list of authors. Inès

${ }^{\star}$ Correspondence: Dr PJK Kuppen; E-mail: P.J.K.Kuppen@lumc.nl

${ }^{4}$ These authors contributed equally to this work.

\section{MiR-224 promotes the chemoresistance of human lung adenocarcinoma cells to cisplatin via regulating G1/S transition and apoptosis by targeting P21WAF1/CIP1}

H Wang, L-J Zhu, Y-C Yang, Z-X Wang and R Wang

Correction to: British Journal of Cancer (2014) 111, 339-354; doi:10.1038/bjc.2014.157

A

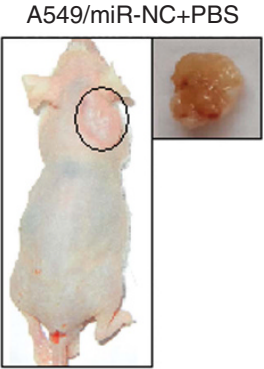

A549/miR-NC+CDDP

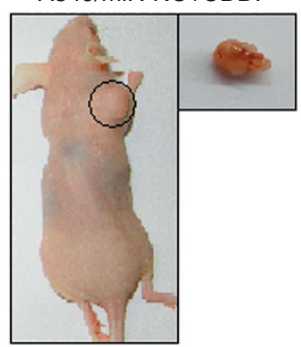

Upon publication of this paper earlier in Volume 111, the authors spotted an error in Figure 7A, where the tumour body diagrams mismatch with the general map of nude mice. The corrected Figure 7A is reproduced below.

Figure 7. Upregulation of miR-224 reduces the in vivo sensitivity of A549 cells to DDP. Mice were treated with DDP $\left(3.0 \mathrm{mg} \mathrm{kg}{ }^{-1}\right.$ body weight; i.p., thrice) or with $0.1 \mathrm{ml} \mathrm{PBS} \mathrm{(pH} \mathrm{7.4;} \mathrm{i.p.,} \mathrm{thrice).} \mathrm{(A)} \mathrm{Representative} \mathrm{features} \mathrm{of} \mathrm{tumours} 28$ days after inoculation using A549/miR-224 or A549/ miR-NC cells treated with PBS or DDP.

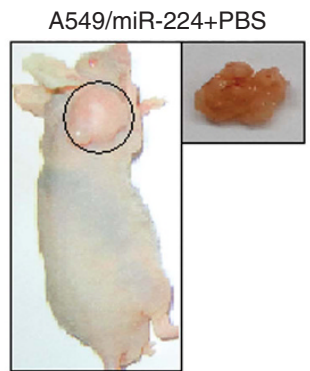

A549/miR-224+CDDP

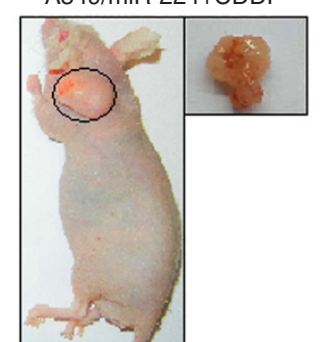

\section{Relationships between pazopanib exposure and clinical safety and efficacy in patients with} advanced renal cell carcinoma

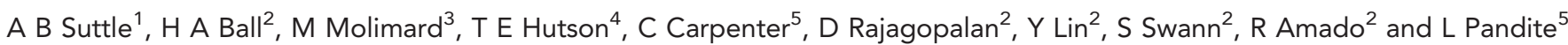
${ }^{1}$ Salix Pharmaceuticals, Inc., 8510 Colonnade Center Drive, Raleigh, NC 27615, USA; ${ }^{2}$ GlaxoSmithKline, 1250S. Collegeville Road, Collegeville, PA 19426, USA; ${ }^{3}$ Pharmacology Department, Université de Bordeaux INSERM U657, 146 Leo Saignat, Bordeaux 33076, France; ${ }^{4}$ GU Oncology Center of Excellence, Baylor University Medical Center, 3410 Worth Street, Dallas, TX 75246, USA and ${ }^{5}$ GlaxoSmithKline, 5 Moore Drive, Research Triangle Park, NC 27709, USA

Correction to: British Journal of Cancer (2014) 111, 1909-1916. doi:10.1038/ bjc. 2014.503

During the final correction process of this paper, the affiliation for Dr AB Suttle was mistakenly applied to two other authors. The full, correct author listing and affiliations are now listed above. The publishers apologise for this error. 